\title{
Effect of putative efflux pump inhibitors and inducers on the antimicrobial susceptibility of Campylobacter jejuni and Campylobacter coli
}

\author{
Correspondence \\ Minna Hannula \\ minna.hannula@helsinki.fi
}

Received 13 December 2007

Accepted 29 February 2008

\author{
Minna Hannula and Marja-Liisa Hänninen
}

\author{
Department of Food and Environmental Hygiene, Faculty of Veterinary Medicine, University of \\ Helsinki, Helsinki, Finland
}

\section{INTRODUCTION}

Campylobacteriosis is the leading cause of bacterial gastroenteritis in industrialized countries (Friedman et al., 2000). Although in most cases the illness is self-limiting, in severe cases and in immunocompromised patients, the use of antimicrobial agents is warranted. However, the rapid increase in resistant strains and the emergence of strains with multiple resistances are of growing concern (Ruiz et al., 2007).

It has been established that efflux pumps, particularly the resistance-nodulation-cell division (RND) type CmeABC pump, have an important role in the antimicrobial resistance of Campylobacter jejuni (Lin et al., 2002). The same efflux pump has recently been characterized in Campylobacter coli (Corcoran et al., 2005). This pump is able to significantly decrease the susceptibility of Campylobacter to a number of antimicrobials (Lin et al., 2002).

Efforts have been made to find substances capable of reversing the action of the efflux pumps and which therefore have the potential to prevent antimicrobial resistance in vivo. A broad-spectrum efflux pump inhibitor (EPI), phenyl-arginine- $\beta$-naphthylamide $(\mathrm{PA} \beta \mathrm{N})$, was first characterized in Pseudomonas aeruginosa (Lomovskaya et al., 2001) and has since been studied in several bacteria, including Campylobacter (Cagliero et al., 2005; Gibreel et al., 2007; Payot et al., 2004). Another putative EPI is 1-

Abbreviations: EPI, efflux pump inhibitor; NMP, 1-(1-naphthylmethyl)piperazine; $\mathrm{PA} \beta \mathrm{N}$, phenyl-arginine- $\beta$-naphthylamide; RND, resistancenodulation-cell division. (1-naphthylmethyl)-piperazine (NMP), characterized in Escherichia coli (Bohnert \& Kern, 2005). It is able to partially reverse multidrug resistance in some members of the Enterobacteriaceae and in Acinetobacter baumannii (Bohnert \& Kern, 2005; Pannek et al., 2006; Schumacher et al., 2006) but its effects on Campylobacter have not been studied previously.

In E. coli, salicylic acid binds to a repressor protein, MarR, which results in the overexpression of the global regulatory gene marA. This causes the down-regulation of the outermembrane protein $\mathrm{OmpF}$ and an increase in the synthesis of the RND multidrug efflux pump AcrAB-TolC, which leads to decreased antimicrobial accumulation (Price et al., 2000). Salicylate has also been reported to increase the effect of antimicrobial aminoglycosides in $E$. coli (Aumercier et al., 1990). Finally, Lin et al. (2005) have shown that bile salts, such as deoxycholate, significantly induce the expression of $c m e A B C$ in $C$. jejuni by preventing the binding of the transcriptional repressor of cme $A B C$ (CmeR). This can lead to increased resistance to several antibiotics (Lin et al., 2005).

The aim of this study was to analyse the effect of these two EPIs, as well as the two putative efflux pump inducers, on the susceptibility of $C$. jejuni and C. coli to erythromycin, ciprofloxacin, kanamycin, tetracycline and rifampicin.

\section{METHODS}

Bacterial strains. Five C. jejuni and four C. coli strains, isolated from poultry, human patients or pigs, were included in this study. The $C$. jejuni strain ATCC 33560 was used as a reference strain. Strain 49/ 
7RAT was a laboratory-derived variant of 49/7R, obtained by selection on media containing increasing concentrations of ampicillin and tetracycline. Also included were ciprofloxacin-resistant derivatives of ATCC 33560, Brt1, B25, B42, B67, 49/7R and 49/7RAT, obtained in vitro by growing the parent strains in media containing $1 \mathrm{mg}$ ciprofloxacin $1^{-1}$ (Hänninen \& Hannula, 2007).

Determination of MICs. MICs of ciprofloxacin, erythromycin, rifampicin and tetracycline (all Sigma-Aldrich) in the presence and

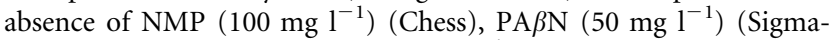
Aldrich), sodium salicylate $\left(100 \mathrm{mg} \mathrm{l}^{-1}\right.$ ) or sodium deoxycholate $\left(1 \mathrm{mg} \mathrm{ml}{ }^{-1}\right.$ ) (both Merck) were determined by using the agar dilution method following the guidelines set by the CLSI (2006). All experiments were repeated at least three times. The growth of each strain was verified on control plates containing the indicated amounts of the studied efflux pump inhibitors and inducers.

\section{RESULTS AND DISCUSSION}

\section{MIC values for the $C$. jejuni and $C$. coli strains and their variants}

The MIC levels were determined for each strain studied and are shown in Table 1. Ciprofloxacin-resistant variants were obtained by selection on media containing $1 \mathrm{mg}$ ciprofloxacin $1^{-1}$ (Hänninen \& Hannula, 2007). The resistant variants had 16- to 128 -fold greater MIC values than the respective parent strains, which corresponded to MIC values from 2 to $64 \mathrm{mg} \mathrm{l}^{-1}$.

\section{Effect of PA $\beta \mathbf{N}$}

In congruence with previous studies (Cagliero et al., 2005; Gibreel et al., 2007; Payot et al., 2004), PA $\beta \mathrm{N}$ at a concentration of $50 \mathrm{mg} \mathrm{l}^{-1}$ resulted in an 8- to 32 -fold decrease in the erythromycin MIC levels, corresponding to MIC values from 0.032 to $1 \mathrm{mg} \mathrm{l}^{-1}$. Our study included only erythromycin-susceptible and low-level resistant strains that most probably lacked the A2075G mutation in the $23 \mathrm{~S}$ rRNA gene associated with high-level macrolide resistance. A previous study, involving high-level resistant strains, showed that when all copies of the target gene carry an A2074C mutation, PA $\beta \mathrm{N}$ is unable to decrease the resistance level, indicating that efflux has little significance in the high-level resistance to erythromycin in these instances (Gibreel et al., 2007).

$\mathrm{PA} \beta \mathrm{N}$ also decreased rifampicin resistance 8- to 64 -fold in the studied strains. C. jejuni and C. coli are intrinsically resistant to rifampicin, and $\mathrm{PA} \beta \mathrm{N}$ 's ability to reduce this resistance suggests that rifampicin resistance in Campylobacter is at least partly associated with efflux. A recently published work on tetracycline reported 1.3- to 2.6-fold decrease in tetracycline resistance in highly resistant $C$. jejuni strains in the presence of $30 \mu \mathrm{g} \mathrm{PA} \beta \mathrm{N}$ $\mathrm{ml}^{-1}$ (Gibreel et al., 2007). Congruently, the two tetracycline-resistant strains (6590 and 49/7RAT CIP) included in our study also had a 2 -fold reduction in the tetracycline MIC values, but the same phenomenon was not observed in tetracycline-susceptible strains. With regards to kanamycin and ciprofloxacin, there was no evidence of PA $\beta \mathrm{N}$ affecting the MICs of these antimicrobials for the bacteria.

The inactivation by insertional mutagenesis of the $c m e B$ gene of $C$. jejuni has led to an 8-fold reduction in ciprofloxacin MIC levels, 4- to 16-fold reduction in erythromycin MIC levels, 64- to 128-fold decrease in rifampicin MIC levels and an 8-fold decrease in tetracycline MIC levels (Lin et al., 2002). The effects on the MIC levels obtained when the PA $\beta \mathrm{N}$ pump inhibitor was added to the growth media were less pronounced or absent, suggesting that the action of $\mathrm{PA} \beta \mathrm{N}$ does not result in the complete inactivation of CmeABC and that this substance may have other modes of action. However, the strong decreasing effect of $\mathrm{PA} \beta \mathrm{N}$ on the MIC levels of erythromycin and rifampicin warrants the use of $\mathrm{PA} \beta \mathrm{N}$ in further studies involving these antimicrobials.

\section{Effect of NMP}

Studies conducted with NMP showed a 2- to 8-fold decrease in erythromycin and rifampicin MIC levels for $C$. jejuni and C. coli, but did not show an increase in the susceptibility of Campylobacter to tetracycline, kanamycin or ciprofloxacin. According to our results, NMP confers an increase in susceptibility to the same antimicrobials as $\mathrm{PA} \beta \mathrm{N}$ but with less effectiveness, which is in agreement with the studies on members of the Enterobacteriaceae and Acinetobacter baumannii (Bohnert \& Kern, 2005; Pannek et al., 2006; Schumacher et al., 2006), indicating that NMP confers effects on MIC levels that differ from those seen with $\mathrm{PA} \beta \mathrm{N}$, suggesting a different mode of action for these pump inhibitors.

\section{Effect of sodium salicylate}

Salicylate and acetylsalicylate are commonly used nonsteroidal anti-inflammatory agents that are often used in combination with antimicrobials for the treatment of microbial diseases. However, salicylate has been shown to decrease the susceptibility of bacteria, such as E. coli, to antimicrobials by altering membrane proteins and inducing efflux pumps (Price et al., 2000). Since C. jejuni possesses an outer-membrane protein (MOMP) belonging to the same porin family as E. coli OmpF (Bolla et al., 1995) and a multidrug efflux pump (CmeABC) belonging to the same RND family as AcrAB-TolC (Lin et al., 2002), it has been speculated that salicylate might also decrease antimicrobial accumulation in C. jejuni. A previous study by Randall et al. (2003) showed a small but significant effect of salicylate on the ciprofloxacin resistance of Campylobacter, and our results confirm a 2- to 4-fold increase in MIC values for ciprofloxacin in almost all strains, with ciprofloxacin-susceptible strains and their respective variants reacting alike. In the study by Randall et al. (2003), no effect was observed on kanamycin MIC levels, which was confirmed by our study, but unlike the 
Table 1. MICs ( $\left.\mathrm{mg} \mathrm{I}^{-1}\right)$ of antimicrobial agents in the presence and absence of PA $\beta \mathrm{N}\left(50 \mathrm{mg} \mathrm{I}^{-1}\right)$, NMP $\left(100 \mathrm{mg} \mathrm{I}^{-1}\right)$, sodium salicylate $\left(100 \mathrm{mg} \mathrm{l}^{-1}\right)$ or sodium deoxycholate $\left(1 \mathrm{mg} \mathrm{ml}^{-1}\right)$

At least 4-fold changes are indicated in bold.

\begin{tabular}{|c|c|c|c|c|c|}
\hline Strain & Ciprofloxacin & Erythromycin & Kanamycin & Rifampicin & Tetracycline \\
\hline ATCC 33560 C. jejuni & 0.25 & 2 & 4 & 512 & 2 \\
\hline$+\mathrm{PA} \beta \mathrm{N}$ & 0.25 & 0.25 & 4 & 64 & 4 \\
\hline + Salicylate & 0.25 & 2 & 4 & 512 & 2 \\
\hline+ Deoxycholate & 0.25 & 2 & 4 & 512 & 2 \\
\hline ATCC 33560 CIP C. jejuni & 16 & 4 & 4 & 512 & 4 \\
\hline + NMP & 8 & 0.5 & 4 & 256 & 1 \\
\hline + Salicylate & 8 & 2 & 4 & 512 & 2 \\
\hline + Deoxycholate & 8 & 4 & 4 & 512 & 4 \\
\hline Brt1 C. jejuni & 0.125 & 1 & 4 & 512 & 1 \\
\hline$+\mathrm{PA} \beta \mathrm{N}$ & 0.125 & 0.125 & 4 & 8 & 1 \\
\hline$+\mathrm{NMP}$ & 0.125 & 0.25 & 4 & 128 & 0.5 \\
\hline + NMP & 4 & 0.25 & 4 & 256 & 0.5 \\
\hline + Salicylate & 16 & 2 & 4 & 1024 & 2 \\
\hline+ Deoxycholate & 8 & 1 & 4 & 1024 & 2 \\
\hline B42 C. jejuni & 0.063 & 0.5 & 4 & 256 & 0.5 \\
\hline$+\mathrm{PA} \beta \mathrm{N}$ & 0.063 & 0.063 & 4 & 8 & 0.5 \\
\hline$+\mathrm{NMP}$ & 0.063 & 0.25 & 4 & 64 & 0.25 \\
\hline + Salicylate & 0.25 & 1 & 4 & 512 & 1 \\
\hline+ Deoxycholate & 0.125 & 1 & 4 & 512 & 1 \\
\hline B42 CIP C. jejuni & 4 & 0.5 & 4 & 256 & 0.5 \\
\hline$+\mathrm{PA} \beta \mathrm{N}$ & 4 & 0.063 & 4 & 8 & 0.5 \\
\hline + NMP & 4 & 0.25 & 4 & 64 & 0.25 \\
\hline B67 CIP C. jejuni & 16 & 1 & 8 & 512 & 0.5 \\
\hline$+\mathrm{PA} \beta \mathrm{N}$ & 16 & 0.125 & 4 & 32 & 0.5 \\
\hline + NMP & 16 & 1 & 8 & 256 & 0.25 \\
\hline + Salicylate & 64 & 1 & 2 & 1024 & 1 \\
\hline + Deoxycholate & 16 & 1 & 8 & 1024 & 0.5 \\
\hline 49/7R C. jejuni & 0.063 & 0.5 & 4 & 1024 & 0.5 \\
\hline$+\mathrm{PA} \beta \mathrm{N}$ & 0.063 & 0.063 & 4 & 64 & 0.5 \\
\hline + NMP & 0.063 & 0.25 & 4 & 512 & 0.25 \\
\hline + Salicylate & 0.25 & 1 & 4 & 2048 & 1 \\
\hline + Deoxycholate & 0.063 & 0.5 & 4 & 2048 & 0.5 \\
\hline 49/7R CIP C. jejuni & 2 & 4 & 4 & 1024 & 4 \\
\hline$+\mathrm{PA} \beta \mathrm{N}$ & 1 & 0.5 & 4 & 128 & 4 \\
\hline$+\mathrm{NMP}$ & 1 & 2 & 4 & 512 & 2 \\
\hline + Salicylate & 2 & 4 & 2 & 1024 & 4 \\
\hline + Deoxycholate & 4 & 4 & 4 & 2048 & 4 \\
\hline 49/7RAT C. jejuni & 1 & 16 & 4 & 512 & 8 \\
\hline$+\mathrm{PA} \beta \mathrm{N}$ & 1 & 1 & 4 & 32 & 8 \\
\hline$+\mathrm{NMP}$ & 0.5 & 4 & 4 & 256 & 4 \\
\hline + Salicylate & 1 & 8 & 4 & 512 & 4 \\
\hline
\end{tabular}


Table 1. cont.

\begin{tabular}{|c|c|c|c|c|c|}
\hline Strain & Ciprofloxacin & Erythromycin & Kanamycin & Rifampicin & Tetracycline \\
\hline + Deoxycholate & 1 & 16 & 4 & 512 & 8 \\
\hline 49/7RAT CIP C. jejuni & 64 & 16 & 4 & 512 & 16 \\
\hline$+\mathrm{PA} \beta \mathrm{N}$ & 32 & 1 & 4 & 64 & 8 \\
\hline$+\mathrm{NMP}$ & 32 & 4 & 4 & 256 & 4 \\
\hline + Salicylate & 32 & 8 & 2 & 512 & 8 \\
\hline + Deoxycholate & 32 & 16 & 4 & 512 & 16 \\
\hline B25 C. coli & 0.063 & 1 & 2 & 128 & 1 \\
\hline$+\mathrm{PA} \beta \mathrm{N}$ & 0.063 & 0.032 & 1 & 4 & 1 \\
\hline$+\mathrm{NMP}$ & 0.063 & 0.125 & 1 & 64 & 1 \\
\hline + Salicylate & 0.25 & 0.5 & 1 & 256 & 1 \\
\hline + Deoxycholate & 0.063 & 1 & 2 & 128 & 1 \\
\hline B25 CIP C. coli & 8 & 1 & 2 & 128 & 0.5 \\
\hline$+\mathrm{PA} \beta \mathrm{N}$ & 4 & 0.032 & 1 & 8 & 1 \\
\hline + NMP & 8 & 0.125 & 1 & 16 & 1 \\
\hline + Salicylate & 16 & 0.5 & 1 & 128 & 2 \\
\hline+ Deoxycholate & 8 & 1 & 2 & 256 & 0.5 \\
\hline S140R C. coli & 0.125 & 1 & 2 & 128 & 1 \\
\hline$+\mathrm{PA} \beta \mathrm{N}$ & 0.125 & 0.063 & 2 & 8 & 0.5 \\
\hline + NMP & 0.125 & 0.5 & 4 & 32 & 0.5 \\
\hline + Salicylate & 0.25 & 1 & 2 & 128 & 1 \\
\hline+ Deoxycholate & 0.125 & 0.5 & 2 & 256 & 1 \\
\hline 72277 C. coli & 0.25 & 1 & 8 & 1024 & 2 \\
\hline$+\mathrm{PA} \beta \mathrm{N}$ & 0.125 & 0.063 & 8 & 64 & 2 \\
\hline$+\mathrm{NMP}$ & 0.125 & 0.25 & 8 & 256 & 1 \\
\hline + Salicylate & 0.5 & 1 & 8 & 1024 & 2 \\
\hline + Deoxycholate & 0.25 & 1 & 8 & 1024 & 2 \\
\hline 6590 C. coli & 0.25 & 2 & 8 & 512 & 64 \\
\hline$+\mathrm{PA} \beta \mathrm{N}$ & 0.25 & 0.125 & 4 & 16 & 32 \\
\hline + NMP & 0.25 & 0.25 & 8 & 256 & 32 \\
\hline + Salicylate & 0.5 & 2 & 8 & 512 & 64 \\
\hline+ Deoxycholate & 0.25 & 2 & 8 & 512 & 64 \\
\hline
\end{tabular}

previous study, we were unable to observe changes in tetracycline or erythromycin MIC levels in the presence of sodium salicylate (Randall et al., 2003). In 7 out of 17 strains, a 2-fold increase in rifampicin resistance was found when salicylate was included in the medium.

\section{Effect of sodium deoxycholate}

In the study by Lin et al. (2005), the expression of cmeABC was significantly induced by various bile salts, such as deoxycholate. Cholate $\left.(1 \mathrm{mg} \mathrm{ml})^{-1}\right)$ and taurocholate (25 mg ml $\mathrm{m}^{-1}$ ) included in the $\mathrm{MH}$ broth produced, respectively, a 0 - to 2 -fold and a 2 - to 4 -fold increase in the MIC levels of various antimicrobials, such as ciprofloxacin and erythromycin, in C. jejuni. In our study, sodium deoxycholate $\left(1 \mathrm{mg} \mathrm{ml}^{-1}\right)$ used in the agar dilution experiments had little effect on the MIC values of the studied antimicrobials. Strains with 2 -fold increases in rifampicin MIC values were variably found in each round of testing, suggesting that although deoxycholate may have had an increasing effect on rifampicin MIC values, this effect was too modest to be confirmed by these experiments.
In summary, $\mathrm{PA} \beta \mathrm{N}$ induced an 8 - to 32 -fold and 8 - to 64 fold reduction in the MIC values of erythromycin and rifampicin for the studied strains, respectively. NMP produced a smaller, 2- to 8 -fold reduction, for the same antimicrobials. With regards to the putative efflux pump inducers, sodium deoxycholate had little or no effect on the MIC values of the studied antimicrobials, whereas sodium salicylate brought about a small (2- to 4-fold) but reproducible increase in the MIC values for ciprofloxacin, and in $7 / 17$ of the studied strains, a 2 -fold increase in the MIC values for rifampicin. These results further confirm the importance of efflux in Campylobacter resistance and highlight the value of agents that affect efflux pumps as tools for research aimed at the inhibition of $\mathrm{CmeABC}$ and the reversal of antimicrobial resistance in Campylobacter.

\section{ACKNOWLEDGEMENTS}

This work was supported by the Finnish Graduate School on Applied Bioscience: Bioengineering, Food \& Nutrition, Environment (ABS), and the Academy of Finland. We are grateful to Urszula Hirvi for her technical assistance. 


\section{REFERENCES}

Aumercier, M., Murray, D. M. \& Rosner, J. L. (1990). Potentiation of susceptibility to aminoglycosides by salicylate in Escherichia coli. Antimicrob Agents Chemother 34, 786-791.

Bohnert, J. A. \& Kern, W. V. (2005). Selected arylpiperazines are capable of reversing multidrug resistance in Escherichia coli overexpressing RND efflux pumps. Antimicrob Agents Chemother 49, 849-852.

Bolla, J. M., Loret, E., Zalewski, M. \& Pagés, J. M. (1995). Conformational analysis of the Campylobacter jejuni porin. J Bacteriol 177, 4266-4271.

Cagliero, C., Mouline, C., Payot, S. \& Cloeckaert, A. (2005). Involvement of the CmeABC efflux pump in the macrolide resistance of Campylobacter coli. J Antimicrob Chemother 56, 948-950.

CLSI (2006). Performance Standards for Antimicrobial Susceptibility Testing. Supplement M100-S16. Wayne, PA: Clinical and Laboratory Standards Institute.

Corcoran, D., Quinn, T., Cotter, L., O'Halloran, F. \& Fanning, S. (2005). Characterization of a $c m e A B C$ operon in a quinolone-resistant Campylobacter coli isolate of Irish origin. Microb Drug Resist 11, 303-308.

Friedman, C. R., Neimann, J., Wegener, H. C. \& Tauxe, R. V. (2000). Epidemiology of Campylobacter jejuni infections in the United States and other industrialized nations. In Campylobacter, 2nd edn, pp. 121138. Edited by I. Nachamkin \& M. J. Blaser. Washington, DC: American Society for Microbiology.

Gibreel, A., Wetsch, N. M. \& Taylor, D. E. (2007). Contribution of the CmeABC efflux pump to macrolide and tetracycline resistance in Campylobacter jejuni. Antimicrob Agents Chemother 51, 3212-3216.

Hänninen, M. L. \& Hannula, M. (2007). Spontaneous mutation frequency and emergence of ciprofloxacin resistance in Campylobacter jejuni and Campylobacter coli. J Antimicrob Chemother 60, 1251-1257.

Lin, J., Michel, L. O. \& Zhang, O. (2002). CmeABC functions as a multidrug efflux system in Campylobacter jejuni. Antimicrob Agents Chemother 46, 2124-2131.
Lin, J., Cagliero, C., Guo, B., Barton, Y. W., Maurel, M. C., Payot, S. \& Zhang, $\mathbf{Q}$. (2005). Bile salts modulate expression of the CmeABC multidrug efflux pump in Campylobacter jejuni. J Bacteriol 187, 74177424.

Lomovskaya, O., Warren, M. S., Lee, A., Galazzo, J., Fronko, R., Lee, M., Blais, J., Cho, D., Chamberland, S. \& other authors (2001). Identification and characterization of inhibitors of multidrug resistance efflux pumps in Pseudomonas aeruginosa: novel agents for combination therapy. Antimicrob Agents Chemother 45, 105-116.

Pannek, S., Higgins, P. G., Steinke, P., Jonas, D., Akova, M., Bohnert, J. A., Seifert, H. \& Kern, W. V. (2006). Multidrug efflux inhibition in Acinetobacter baumannii: comparison between 1-(1-naphthylmethyl)piperazine and phenyl-arginine-beta-naphthylamide. J Antimicrob Chemother 57, 970-974.

Payot, S., Avrain, L., Magras, C., Praud, K., Cloeckaert, A. \& ChaslusDancla, E. (2004). Relative contribution of target gene mutation and efflux to fluoroquinolone and erythromycin resistance, in French poultry and pig isolates of Campylobacter coli. Int J Antimicrob Agents 23, 468-472.

Price, C. T., Lee, I. R. \& Gustafson, J. E. (2000). The effects of salicylate on bacteria. Int J Biochem Cell Biol 32, 1029-1043.

Randall, L. P., Ridley, A. M., Cooles, S. W., Sharma, M., Sayers, A. R., Pumbwe, L., Newell, D. G., Piddock, L. J. \& Woodward, M. J. (2003). Prevalence of multiple antibiotic resistance in 443 Campylobacter spp. isolated from humans and animals. J Antimicrob Chemother 52, 507510 .

Ruiz, J., Marco, F., Oliveira, I., Vila, J. \& Gascon, J. (2007). Trends in antimicrobial resistance in Campylobacter spp. causing traveler's diarrhea. APMIS 115, 218-224.

Schumacher, A., Steinke, P., Bohnert, J. A., Akova, M., Jonas, D. \& Kern, W. V. (2006). Effect of 1-(1-naphthylmethyl)-piperazine, a novel putative efflux pump inhibitor, on antimicrobial drug susceptibility in clinical isolates of Enterobacteriaceae other than Escherichia coli. $J$ Antimicrob Chemother 57, 344-348. 